\title{
BMJ Open Critical appraisal of clinical practice guidelines for the diagnosis and treatment of stress urinary incontinence using AGREE II instrument: a systematic review protocol
}

Xiaowei Yang, ${ }^{\odot 1,2}$ AiXia Zhang, ${ }^{3}$ Yanquan Tan, ${ }^{4}$ Zhengbao Zhang, ${ }^{5}$ Jie Zhang $^{2}$

To cite: Yang $X$, Zhang $A X$, Tan Y, et al. Critical appraisal of clinical practice guidelines for the diagnosis and treatment of stress urinary incontinence using AGREE II instrument: a systematic review protocol. BMJ Open 2019;9:e030299. doi:10.1136/ bmjopen-2019-030299

- Prepublication history and additional material for this paper are available online. To view these files, please visit the journal online (http://dx.doi org/10.1136/bmjopen-2019030299).

Received 31 March 2019 Revised 23 August 2019 Accepted 29 August 2019
Check for updates

(C) Author(s) (or their employer(s)) 2019. Re-use permitted under CC BY-NC. No commercial re-use. See rights and permissions. Published by BMJ.

For numbered affiliations see end of article.

Correspondence to

Xiaowei Yang;

xiaowei.yang@kcl.ac.uk

\section{ABSTRACT}

Introduction Stress urinary incontinence is a major health problem, and several clinical guidelines have been formulated and released regarding this in different countries. However, the recommendations in these guidelines formulated by different organisations and countries are inconsistent. This review aims to conduct a critical appraisal of clinical practice guidelines for the diagnosis and treatment of stress urinary incontinence. Methods and analysis We will conduct a comprehensive search in the following databases: PubMed, Embase, Medline, Cochrane Library, three Chinese databases and six guideline databases. The databases will be searched from January 2003, and the comprehensive search will be done again to include all the qualified guidelines before making conclusions. The quality of clinical practice guidelines will be assessed by three appraisers using the Appraisal of Guidelines Research and Evaluation II instrument, and this will be scored. The recommendation available in the guidance will also be summarised in different domains including the diagnosis standard, recommended examination and questionnaire for assessment, conservative treatment and surgical treatment.

Ethics and dissemination This review will be disseminated through peer-reviewed publications. The results will help inform the health practitioners about the recommendations in clinical practice guidelines.

PROSPERO registration number CRD42018115743.

\section{INTRODUCTION}

Urinary incontinence is defined by the International Continence Society (ICS) as 'complaint of involuntary loss of urine'. It commonly affects women, especially the aged. The prevalence of urinary incontinence worldwide ranges from $5 \%$ to $69 \%$ and is influenced by different definitions, populations and standard of diagnosis. ${ }^{2}$ It is reported that more than 20000000 women suffer from incontinence in the USA. ${ }^{3}$ In addition, $30.9 \%$ of women experienced urinary incontinence in China according to
Strengths and limitations of this study

- We will apply Appraisal of Guidelines Research and Evaluation (AGREE) II instrument, which is validated and well established in guideline appraisal.

- This proposed review will involve an extensive literature search and a rigorous appraisal process, which will include 13 databases of different countries.

- We will provide an extensive training to the reviewers on how to use the AGREE II tool.

- The clinical practice guidelines that will be included in this review are in English and Chinese.

- This review will not include grey literatures that are not indexed in the databases.

a survey conducted in 2009. ${ }^{4}$ Urinary incontinence has many adverse effects on social interactions and activities, physical exercises and sexual relationships, although it does not endanger the lives of patients. ${ }^{5}$ For example, Saadoun et al found that patients with urinary incontinence tend not to go out because of the fear of urine leakage. ${ }^{7}$ Besides, it was confirmed that the most frequent effects of urinary incontinence are emotional disturbance like avoidance, social embarrassment and limiting behaviour. ${ }^{8}$ Urinary incontinence has different subtypes including stress incontinence, urgency incontinence, postural incontinence, nocturnal enuresis, mixed incontinence, continuous incontinence, insensible incontinence and coital incontinence. ${ }^{9}$ There are three main subtypes: stress urinary incontinence (SUI), urgency urinary incontinence and mixed urinary incontinence. ${ }^{9}$ According to the standard definition from ICS, SUI is defined as 'complaint of involuntary loss of urine on effort or physical exertion including sporting activities, sneezing, or coughing'. ${ }^{1}$ SUI has 
the highest prevalence among these types. ${ }^{10}$ The prevalence of SUI is as high as $18.9 \%$ in China. ${ }^{4}$

Clinical guidelines are evidence-based documents, which are important for clinical practitioners to deliver high-quality care to patients, as well as improve patient prognosis. ${ }^{11} 12$ A large number of countries have developed their national guidance for the diagnosis and treatment of urinary incontinence. ${ }^{13-17}$ Additionally, guidelines for SUI have been published by different academies including urology and obstetrics and gynaecology.

However, the quality of the guidelines for urinary incontinence varies greatly although there are various guidance documents available. ${ }^{18}{ }^{19}$ Research showed that the published Chinese clinical practice guidelines are of poor quality. The guidelines in China are below the international standard although progress has been made over the past years. It is reported that low-quality clinical guidelines have an adverse effect on the compliance of healthcare providers and the prognosis of patients. ${ }^{20}$ Besides, the use of guidelines greatly depends on the implementation and dissemination process even if they are well developed. ${ }^{21}{ }^{22}$ It is reported that insufficient time and staffs, shortage of diagnostic tools and competences and low enthusiasm when treating patients are the most influential reasons for low adherence to the guidelines. These problems are commonly found in many countries and indicate that the implementation of guidelines and treatment of urinary incontinence require both primary and secondary care. ${ }^{23} 24$ Appraisal of Guidelines for Research and Evaluation (AGREE) is a tool widely used to assess the transparency and the methodological rigour of guideline development. ${ }^{25}$ In 2009, AGREE II was developed to improve measurement properties. AGREE II will be used in this research to assess the quality of available clinical guidelines on urinary incontinence, and it may provide suggestions for further development of clinical guidelines in China.

\section{Objectives}

The aim of this study is to investigate the quality of published clinical guidelines for the diagnosis and treatment of SUI using the AGREE II.

\section{METHODS AND ANALYSIS}

\section{Protocol and registration}

This systematic review will be conducted according to the Preferred Reporting Items for Systematic Review and Meta-Analyses (PRISMA), ${ }^{26}$ and this protocol was written according to the PRISMA protocol checklist which can be found as online supplementary file 1 .

\section{Literature search}

We will conduct a comprehensive literature search in the following databases: PubMed, Embase (using the Ovid interface), Medline, National Guideline Clearinghouse (NGC), the National Institute for Health and Care Excellence, Cochrane Library, Australian Clinical Practice
Box 1 Sample search strategy for Ovid Embase

1. $\exp$ Stress Urinary Incontinence/

2. exp Urinary Incontinence/

3. one or 2

4. exp guideline/

5. exp consensus/

6. exp recommendation/

7. exp policy/

8. four or five or six or 7

9. 3 and 8

Guidelines, Scottish Intercollegiate Guidelines Network, Canadian Agency for Drugs and Technologies in Health, the Guidelines International Network, China National Knowledge Infrastructure, Chinese Biomedical Literature Database and Wanfang database. The sample search is shown in box 1 .

\section{Inclusion criteria and exclusion criteria}

We will include clinical guidelines, which have recommendations for patient care on SUI. Clinical guidelines and consensus statements for the diagnosis and/or treatments of SUI will be included if they (1) were published from January 2003, and the comprehensive search will be done again to include all the qualified guidelines before making conclusions; (2) were written in English or Chinese and (3) provided clinical guidelines or recommendations for the diagnosis and treatment of SUI.

The exclusion criteria are as follows: (1) former documents replaced by updated versions from the same organisation; (2) designed documents for local use; the full text is unavailable or only abstract is available and (3) comments on the existing guidelines.

\section{Guideline selection and data extraction}

There will be two reviewers to screen the titles and abstracts independently based on the eligibility criteria. The two reviewers will report the reasons for exclusion if documents are excluded. If there is a disagreement, it will be resolved through a discussion with a third reviewer.

Records of the guidelines will be added to an Excel worksheet including document characteristics (year of publication and update, first author and the number of authors and country), methods of grading evidence, methods of formulating recommendation and scope of the guideline.

\section{Assessment and training}

AGREE II is used to assess the quality of each guideline or consensus, and the Chinese version of AGREE II, which has been translated and validated, will be used in this study. ${ }^{28}$ AGREE II comprises six domains, which are refined to 23 items. The six domains include scope and purpose, stakeholder involvement, rigour of development, clarity of presentation, applicability and editorial independence. Each domain has different number of items. There are three items each for scope and 
Maximum score $=7($ maximum score of each item $) \times$ the number of items $\times$ the number of appraisers.

Minimum score $=1($ minimum score of each item $) \times$ the number of items $\times$ the number of appraisers.

standardized percentage of each domain=(obtained score- minimum score) $/($ maximum score - minimum score $) \times 100 \%$

Figure 1 The method of scoring.

purpose, stakeholder involvement and clarity of presentation. Rigour of development, applicability and editorial involvement have eight items, four items, and two items, respectively. Each item is rated by reviewers on a 7-point Likert Scale, in which 1 point represents strongly disagree and 7 points represent strongly agree. The score of the individual items in each domain will be calculated as a sum, and a standardised percentage will be scaled (figure 1). ${ }^{29} 30$

Three reviewers involved will be trained to use the AGREE II instrument by studying the AGREE II user's

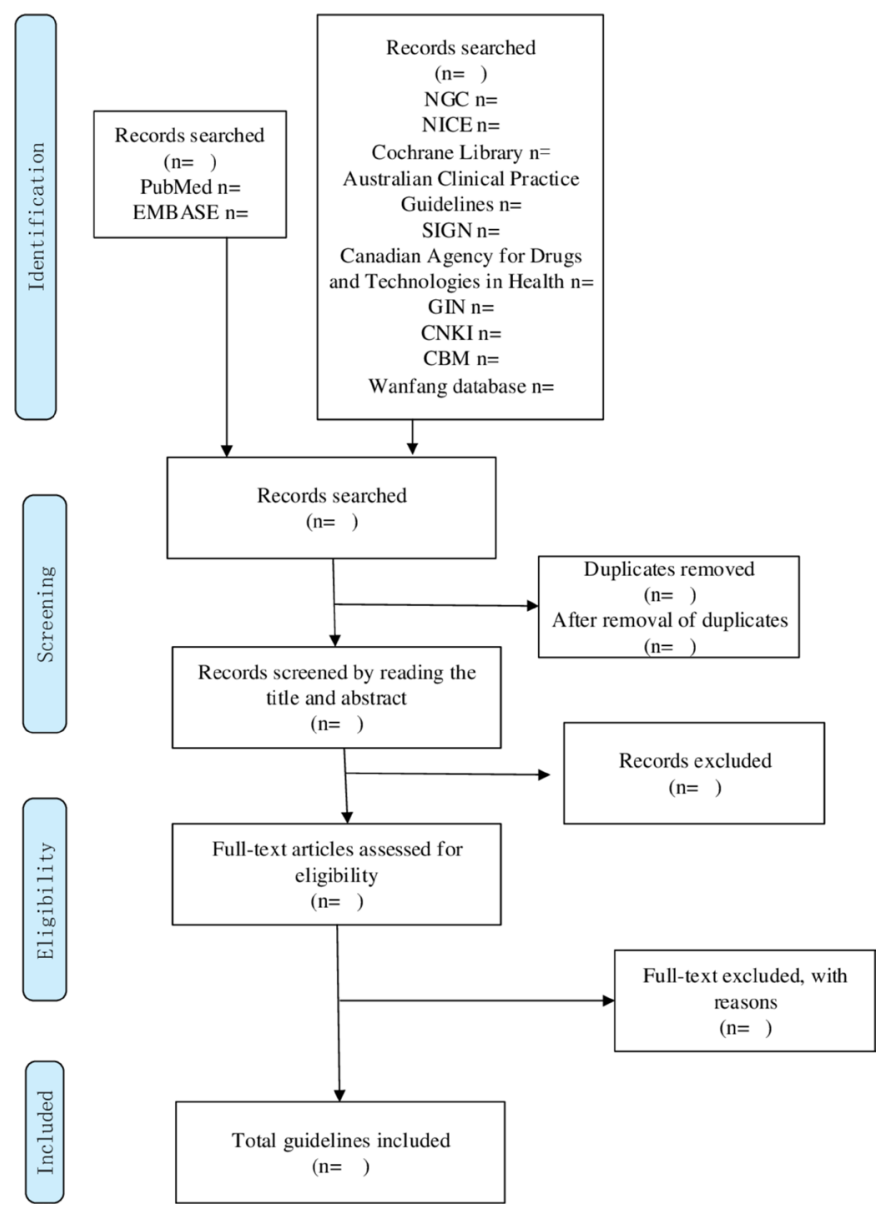

Figure 2 PRISMA flow diagram. CBM, Chinese Biomedical Literature database; CNKI, China National Knowledge Infrastructure; GIN, Guidelines International Network; NGC, National Guideline Clearinghouse; NICE, National Institute for Health and Care Excellence; PRISMA, Preferred Reporting Items for Systematic Reviews and Meta-Analyses; SIGN, Scottish Intercollegiate Guidelines Network. manual and through online training available on My AGREE PLUS platform. Next, a meeting will be held for reviewers who will appraise two clinical guidelines of different quality, and the results will be discussed with each other. The agreement between different reviewers will be calculated by intraclass correlation (ICC) via IBM SPSS 16.0. The score of ICC ranges from 0 to 1 . The closer the score is to 1 , the smaller the variation between different appraisers, and an ICC $\geq 0.70$ is considered acceptable.

\section{Data synthesis}

The study selection process will be performed in accordance with PRISMA, and will be presented in a flow diagram (figure 2).

We will manually extract the data and the AGREE scores and present them in a descriptive table. The recommendation available in the guidance will be summarised in domains relating to the diagnosis and treatment of SUI, including the diagnosis standard, recommended examination and questionnaire for assessment, conservative treatment and surgical treatment.

\section{Patient and public involvement}

No patients were involved in this systematic review.

\section{DISCUSSION}

Updating clinical practice guidelines (CPGs) highly rely on the topic because development in some fields is very rapid and new evidence should be considered when developing CPGs. It is estimated by Shekelle et al that $50 \%$ of the CPGs will be outdated after 5.8 years and $10 \%$ of CPGs are obsolete after 3.6 years. ${ }^{31}$ The main purpose of this systematic review is to assess the quality of CPGs on SUI from both foreign countries and China and provide suggestions for further development of CPGs. For the purpose and the feasibility of our study, there are two reasons for limiting the time to January 2003. First, considering most guidelines updated in recent years, the updated guidelines will be included in this systematic review instead of the former documents. Second, if the guidelines were developed long time ago and do not have any updates, they might be less instructive in developing new guidelines than the updated version.

We expect to appraise the quality of clinical guidelines for SUI using the AGREE II instrument through this proposed study. Besides, we will provide a summary of guideline recommendations on SUI. To date, no appraisal for the quality of clinical guidelines for SUI reported in English and Chinese.

The limitations of this systematic review might be the language restriction on the guidelines and the exclusion of clinical guidelines published in other databases. Second, there might be a publication bias because guidelines on poor quality might not be included in the databases we searched. However, we considered that the publication bias might be minimal because previous studies showed that the quality of clinical guidelines varies widely. 
In conclusion, there is a need to critically appraise and systematically review clinical practice guidelines for the diagnosis and treatment of SUI. This protocol of systematic review provides a structured and rigorous procedure for quality evaluation of clinical guidelines and consensus statements. This review will help the clinical professionals to understand better the quality of guidelines of SUI in China and the recommendation in the guidelines, which may improve the care of SUI. Besides, this review will help the professionals to identify the gap that can be improved when developing a new or updated guideline.

\section{Author affiliations}

${ }^{1}$ King's College London, Florence Nightingale Faculty of Nursing, Midwifery and Palliative care, London, UK

${ }^{2}$ Nursing department, Nanjing Vocational Health College, Nanjing, China

${ }^{3}$ Nursing department, Nanjing Maternity and Child Health Care Hospital, Nanjing, China

${ }^{4}$ Rehabilitation department, Jiangsu Health Vocational and Technical School, Nanjing, China

${ }^{5}$ Teaching and Research Division, Jiangsu Health Higher Vocational and Technical School, Nanjing, China

Acknowledgements The authors thank the reviewers of the manuscript for their critical comments.

Contributors YX conceived this study. YX, ZA and TY designed the inclusion/ exclusion criteria and the searching resource and strategy. YX, ZA, TY, ZZ and ZJ designed the appraisal strategy of each included guideline and consensus. YX, ZA and TY drafted the protocol. ZA and YX contributed to the revision of the manuscript in responding to editor and reviewers. All authors discussed actively in the protocol of the study. YX and ZA contributed equally to this paper.

Funding The authors have not declared a specific grant for this research from any funding agency in the public, commercial or not-for-profit sectors.

Competing interests None declared.

Patient consent for publication Not required.

Provenance and peer review Not commissioned; externally peer reviewed.

Open access This is an open access article distributed in accordance with the Creative Commons Attribution Non Commercial (CC BY-NC 4.0) license, which permits others to distribute, remix, adapt, build upon this work non-commercially, and license their derivative works on different terms, provided the original work is properly cited, appropriate credit is given, any changes made indicated, and the use is non-commercial. See: http://creativecommons.org/licenses/by-nc/4.0/.

\section{REFERENCES}

1. D'Ancona C, Haylen B, Oelke M, et al. The International continence Society (ICS) report on the terminology for adult male lower urinary tract and pelvic floor symptoms and dysfunction. Neurourol Urodyn 2019;38:433-77.

2. Buckley BS, Lapitan MC. Epidemiology Committee of the fourth International consultation on incontinence P. prevalence of urinary incontinence in men, women, and children-current evidence: findings of the fourth International consultation on incontinence. Urology 2010;76:265-70.

3. Landefeld CS. National Institutes of health state-of-the-science conference statement: prevention of fecal and urinary incontinence in adults. Ann Intern Med 2008;148:449-58.

4. Zhu Lan LJ, Chunyan L, Xingming L, et al. Epidemiological study on the prevalence of urinary incontinence in adult women in China. Chinese Journal of Obstetrics and Gynecology 2009;44:776-9.

5. Sen I, Onaran M, Aksakal N, et al. The impact of urinary incontinence on female sexual function. Adv Ther 2006;23:999-1008.

6. Sensoy N, Dogan N, Ozek B, et al. Urinary incontinence in women: prevalence rates,risk factors and impact on quality of life. Pak J Med Sci 2013;29:818-22.
7. Saadoun K, Ringa V, Fritel X, et al. Negative impact of urinary incontinence on quality of life, a cross-sectional study among women aged 49-61 years enrolled in the GAZEL cohort. Neurourol Urodyn 2006;25:696-702.

8. Monz B, Pons ME, Hampel C, et al. Patient-Reported impact of urinary incontinence-Results from treatment seeking women in 14 European countries. Maturitas 2005;52(Suppl 2):24-34.

9. Haylen BT, de Ridder D, Freeman RM, et al. An International Urogynecological Association (IUGA)/International Continence Society (ICS) joint report on the terminology for female pelvic floor dysfunction. Int Urogynecol J 2010;21:5-26.

10. Harvie HS, Shea JA, Andy UU, et al. Validity of utility measures for women with urge, stress, and mixed urinary incontinence. Am J Obstet Gynecol 2014;210:85.e1-6.

11. Davis DA, Taylor-Vaisey A. Translating guidelines into practice. A systematic review of theoretic concepts, practical experience and research evidence in the adoption of clinical practice guidelines. CMAJ 1997;157:408-16.

12. Woolf $\mathrm{SH}$, Grol R, Hutchinson A, et al. Clinical guidelines: potential benefits, limitations, and harms of clinical guidelines. $B M J$ 1999;318:527-30.

13. Thüroff JW, Abrams $\mathrm{P}$, Andersson $\mathrm{K}-\mathrm{E}$, et al. EAU guidelines on urinary incontinence. Eur Urol 2011;59:387-400.

14. Kobashi KC, Albo ME, Dmochowski RR, et al. Surgical treatment of female stress urinary incontinence: AUA/SUFU guideline. $J$ Urol 2017;198:875-83.

15. Bernards ATM, Berghmans BCM, Slieker-Ten Hove MCP, et al. Dutch guidelines for physiotherapy in patients with stress urinary incontinence: an update. Int Urogynecol J 2014;25:171-9.

16. Takahashi S, Takei M, Nishizawa O, et al. Clinical guideline for female lower urinary tract symptoms. Low Urin Tract Symptoms 2016;8:5-29.

17. Obstetrics CAoga. Guidelines for the diagnosis and treatment of stress urinary incontinence in women (2017). Chinese Journal of Obstetrics and Gynecology 2017;52:289-93.

18. Irving G. The agree reporting checklist is useful for assessing the quality of clinical practice Guideline development. BMJ 2016;353.

19. Yao S, Wei D, Chen Y-L, et al. Quality assessment of clinical practice guidelines for integrative medicine in China: a systematic review. Chin J Integr Med 2017;23:381-5.

20. Dang W, Wang XQ, Qiong-Fang WU, et al. Quality evaluation on Chinese clinical practice guidelines in 2011. Chinese Journal of Evidence-Based Medicine 2013;13:760-3.

21. Neta G, Glasgow RE, Carpenter CR, et al. A framework for enhancing the value of research for dissemination and implementation. Am J Public Health 2015;105:49-57.

22. Graham ID, Logan J, Harrison MB, et al. Lost in knowledge translation: time for a MAP? J Contin Educ Health Prof 2006;26:13-24.

23. Albers-Heitner P, Berghmans B, Nieman F, et al. Adherence to professional guidelines for patients with urinary incontinence by general practitioners: a cross-sectional study. J Eval Clin Pract 2008;14:807-11.

24. Kirby M, Artibani W, Cardozo L, et al. Overactive bladder: the importance of new guidance. Int J Clin Pract 2006;60:1263-71.

25. Brouwers MC, Kho ME, Browman GP, et al. Agree II: advancing Guideline development, reporting, and evaluation in health care. Prev Med 2010;51:421-4.

26. Shamseer L, Moher D, Clarke M, et al. Preferred reporting items for systematic review and meta-analysis protocols (PRISMA-P) 2015: elaboration and explanation. BMJ 2015;349:97647.

27. Moher D, Shamseer L, Clarke M, et al. Preferred reporting items for systematic review and meta-analysis protocols (PRISMA-P) 2015 statement. Syst Rev 2015;4:1.

28. Wei Dang WC, Xiaojuan X, Yaolong C, et al. Interpretation of appraisal of guidelines for research and evaluation (agree) tool. Chinese Journal of Evidence Based Pediatrics 2013;8:316-9.

29. Romeo V, Stanzione A, Cocozza S, et al. A critical appraisal of the quality of head and neck cancer imaging guidelines using the agree II tool: a EuroAIM initiative. Cancer Med 2019;8:209-15.

30. Li Q, Li X, Kwong JS-W, et al. Diagnosis and treatment for hyperuricaemia and gout: a protocol for a systematic review of clinical practice guidelines and consensus statements. BMJ Open 2017;7:e014928.

31. Shekelle PG, Ortiz E, Rhodes S, et al. Validity of the agency for healthcare research and quality clinical practice guidelines: how quickly do guidelines become outdated? JAMA 2001;286:1461-7. 